Les ANNALES Les Annales de droit

DE DROIT

12 | 2018

Varia

\title{
Ménager l'âne et le bœuf : retour sur la neutralité du service public
}

Spare the ox and the donkey: the return of the civil service to neutrality

\section{Benoit Camguilhem}

\section{OpenEdition}

Journals

Édition électronique

URL : http://journals.openedition.org/add/1057

DOI : 10.4000/add. 1057

ISSN : 2606-1988

Éditeur

Presses universitaires de Rouen et du Havre

Édition imprimée

Pagination : 123-140

ISBN : 979-10-240-0937-7

ISSN : 1955-0855

Référence électronique

Benoit Camguilhem, " Ménager l'âne et le bœuf : retour sur la neutralité du service public », Les

Annales de droit [En ligne], 12 | 2018, mis en ligne le 05 février 2019, consulté le 05 décembre 2019.

URL : http://journals.openedition.org/add/1057 ; DOI : 10.4000/add.1057

Presses universitaires de Rouen et du Havre 


\section{Ménager l'âne et le bœuf : retour sur la neutralité du service public}

Benoit Camguilhem

La neutralité du service public est une des règles parmi les mieux établies du droit administratif ${ }^{1}$. L'une de celles dont la portée est la plus simple à déterminer. Si certaines questions demeurent complexes, comme celle des repas confessionnels en prison ${ }^{2}$, la neutralité des bâtiments publics est sans doute la dimension de la neutralité la plus évidente à mettre en œuvre. Un mur blanc, sans signe distinctif, suffit à ce que cette obligation soit remplie. L'interdiction d'un drapeau portant une revendication politique sur le fronton d'une mairie ${ }^{3}$, de nommer un établissement scolaire " Jack-Lang ${ }^{4}$ », d'accrocher le portrait du maréchal Pétain au mur de la salle des délibérations d'une mairie ${ }^{5}$,

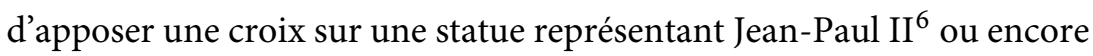
l'interdiction de laisser un crucifix dans la salle du conseil municipal sont autant d'illustrations classiques de cette exigence de neutralité du service public ${ }^{7}$.

Louis Rolland, qui n'en a pas fait l'une des lois du service public, rappelait avec force que «tous les services publics sont neutres ${ }^{8} »$. La neutralité est, en effet, l'une des grandes règles applicables à l'ensemble des services publics, la jurisprudence imposant le «respect de la neutralité

1. Antoine Louvaris, «Le principe de neutralité des services publics. Éléments pour une synthèse », thèse, Paris II, 1995, $674 \mathrm{p}$.

2. CE, 16 juill. 2014, $\mathrm{n}^{\circ}$ 377145, AJDA 2014, p. 2321, note Pierre-Henri Prélot.

3. CE, 27 juill. 2005, Commune de Sainte Anne, rec. 347 ; AJDA 2006, p. 196, note Jean-Baptiste Darracq, DA 2005, comm. 165, note Lucienne Erstein, RFDA 2005, p. 1137, concl. Francis Donnat.

4. TA Lille, 18 déc. 2007, Desurmont.

5. TA Caen, 26 oct. 2010, $\mathrm{n}^{\circ}$ 1000282, préfet du Calvados, JCP A 2010, 2347, note Jean-Paul Markus; DA févr. 2011, comm. 16, Arnaud Duranthon.

6. CE, 25 oct. 2017, Fédération morbihannaise de la libre pensée et autres, $\mathrm{n}^{\circ} 396990$.

7. CAA Nantes, 4 févr. 1999, Association civique Joué Langueurs et autres, rec. 498.

8. Louis Rolland, «Chronique administrative», RDP 1914, p. 412. 
des administrations publiques ${ }^{9} »$ ou, plus généralement, «la neutralité du service public ${ }^{10} »$. Mais, pour bien percevoir le sens et la portée de cette obligation, il est impératif de ne pas omettre qu'il ne s'agit pas d'un principe autonome, mais uniquement d'un corollaire du principe d'égalité $^{11}$. La neutralité du service public n'est pas une fin en soi. Elle n'est ni une valeur ni un but à atteindre. Elle est une condition de l'effectivité du principe d'égalité, car elle garantit l'absence de différenciation ${ }^{12}$. Le principe de neutralité renvoie ainsi à « une obligation s'imposant à l'État qui ne doit favoriser ni ne désavantager, par quelque biais que ce soit, une des conceptions du bien présentes dans la société ${ }^{13}$ ». La neutralité du service public est le moyen par lequel l'égalité de tous les usagers devant le service public peut se réaliser. La neutralité des bâtiments du service public n'est donc ni un combat idéologique ni une lutte théologique. Elle est le moyen le plus simple de permettre que nul, dans le champ du service public, ne soit exclu. Jean Rivero notait ainsi que les bâtiments des services publics doivent se présenter «de telle sorte que leur accès n'entraîne aucune contrainte pour aucune famille d'esprit et que les agents s'interdisent toute discrimination à base idéologique ${ }^{14} »$. Il s'agit bien d'une «neutralité idéologique ${ }^{15}$ ». La stricte neutralité étant «la première garantie de l'égalité des usagers ${ }^{16}{ }$. L'exigence de neutralité

9. CE, 6 juin 1947, Union catholique des hommes du diocèse de Versailles, rec. 251; CE, $1^{\text {er }}$ avr. 1949, Chaveau, rec. 161, D. 1949, jurispr. 532, concl. François Gazier.

10. CE, 13 déc. 1985 , Confédération nationale des groupes autonomes de l'enseignement public, rec. 78 .

11. Il s'agit notamment de la position retenue par le Conseil constitutionnel. Cons. const., 18 sept. 1986, $\mathrm{n}^{\circ}$ 86-217 DC, Loi relative à la liberté de communication, rec.141, AJDA 1987, p. 102, note Patrick Wachsmann. Le Conseil constitutionnel en fait toutefois un "principe fondamental régissant les services publics». Cons. const., 23 juill. 1996, $\mathrm{n}^{\circ}$ 96-380 DC. Une large part de la doctrine se range à cette analyse : Jacques Moreau, Droit administratif, Paris, PUF, 1990, p. 346; Jacques Petit et Pierre-Laurent Frier, Droit administratif, Paris, Montchrestien, 2015, p. 275 ; Benoit Plessix, Droit administratif général, Paris, LexisNexis, 2016, p. 900.

12. Voir René Chapus, Droit administratif général, t. 1, Paris, Montchrestien, 2001, p. 609 et suiv.

13. David Koussens, L'épreuve de la neutralité : la laïcité française entre droits et discours, Bruxelles, Bruylant, 2015, p. 8.

14. Jean Rivero, Les libertés publiques, Paris, PUF, 1989, p. 154.

15. Frédérique de la Morena, Les frontières de la laïcité, Paris, Lextenso, 2016, p. 47.

16. Clément Benelbaz, Le principe de laïcité en droit public français, Paris, L'Harmattan, 2011, p. 269. 
concerne tout type d'expression: politique, philosophique, commerciale $^{17}$ ou religieuse ${ }^{18}$. Elle concerne également tous les bâtiments et tous les services publics, bien que cette exigence soit initialement née dans le domaine scolaire à travers le "principe de la neutralité scolaire en matière religieuse ${ }^{19} »$ ou la «neutralité de l'enseignement ${ }^{20} »$.

En méconnaissance de cette essence de la neutralité du service public, un certain nombre de maires, essentiellement à partir de la fin des années 2000, et le plus souvent à grand renfort médiatique, ont installé des crèches de la nativité dans les halls de leurs mairies au moment des fêtes de Noël. Des crèches existaient sans doute dans des locaux municipaux depuis longtemps, essentiellement dans des villages ou de petites villes et dans certaines régions de France. Mais l'activisme politique sur cette question est récent et s'est prolongé au contentieux devant les juridictions administratives.

La première phase contentieuse a été celle des divergences, les juridictions du fond retenant successivement des solutions contradictoires à compter du début des années 2010. Le tribunal administratif d'Amiens avait considéré qu'une crèche était « un emblème religieux de la religion chrétienne " pour conclure à sa contrariété avec l'article 28 de la loi de $1905^{21}$. Le tribunal administratif de Montpellier avait, de son côté, jugé légale l'installation d'une crèche par la mairie de Béziers au motif qu'elle s'inscrivait dans le cadre d'une exposition ${ }^{22}$. Le tribunal administratif d'Amiens avait également reconnu la légalité de l'installation

17. À titre d'exemple l'organisation d'un concours par une banque dans un établissement scolaire contrevient au principe de neutralité: TA Cergy-Pontoise, $1^{\mathrm{er}}$ juill. 2004, $\mathrm{n}^{\mathrm{o}} 0007594$, Gilbert M.

18. Ce n'est pas directement la laïcité qui s'impose aux services publics, mais la neutralité religieuse doit être vue comme une manifestation concrète de la laïcité dans le champ des services publics, «la laïcité s'efface en réalité derrière le principe plus spécifique de neutralité» (Benoit Plessix, op. cit., p. 899). Par effet de balancier, il est également exact que «la neutralité n'épuise pas à elle seule toute la signification de la laïcité ». Voir Olivier Schrameck, "Laïcité, neutralité et pluralisme», dans Mélanges Jacques Robert, Paris, Montchrestien, 1998, p. 195.

19. TC, 2 juin 1908, Girodet c. Morizot, rec. 597 concl. Tardieu, S. 1908, III, 81, note Maurice Hauriou; CE, 20 janv. 1911, Sieur Porteret, rec. 69, concl. Pichat; CE, 8 avr. 1911, Rosselin, rec. 480; CE, 8 déc. 1911, Dame Rousseaux, rec. 1168; CE, 14 janv. 1916, Association des pères de famille de Gamarde les bains, rec. 30, RDP 1916, p. 52, concl. Corneille, note Gaston Jèze.

20. CE, 30 avr. 1909, Commune de Saint-Memmie, rec. 432.

21. TA Amiens, 30 nov. 2010, Debaye, $\mathrm{n}^{\mathrm{o}}$ 0803521, AJDA 2011, p. 471.

22. TA Montpellier, 16 juill. 2015, M. D., Ligue des droits de l'homme, $\mathrm{n}^{\mathrm{o}} 1405625$, AJDA 2015, p. 1446. 
d'une crèche sur la place Jeanne-Hachette à Beauvais au motif qu'installée "hors de tout contexte religieux et de manière non ostentatoire " elle devait «être regardée comme constituant une des décorations festives traditionnellement associées à Noël; qu'elle ne constitue pas un emblème religieux ${ }^{23}$ ». L'installation de ces crèches n'est pas censurée soit parce qu'elles sont rattachées à une exposition, exception prévue par la loi; soit parce qu'elles sont considérées au regard du contexte comme n'étant pas des signes religieux, écartant de ce fait l'application de l'article 28 de la loi de 1905.

Les divergences sont apparues à travers deux affaires concomitantes. D'abord celle concernant la crèche qui, chaque année depuis plus de trente ans, était installée en fin d'année dans l'hôtel du département de la Vendée. Le tribunal administratif de Nantes annule cette installation au motif que cette représentation est un "emblème religieux spécifique " et qu'aucun particularisme local ne justifie qu'elle soit installée dans un bâtiment du service public ${ }^{24}$. La cour administrative d'appel de Nantes annule le jugement du tribunal au motif que "compte tenu de sa faible taille, de sa situation non ostentatoire et de l'absence de tout autre élément religieux, elle s'inscrit dans le cadre d'une tradition relative à la préparation de la fête familiale de Noël et ne revêt pas la nature d'un "signe ou emblème religieux ${ }^{25}$ " ». La crèche de l'hôtel de ville de Melun a donné une autre occasion aux juridictions du fond de rendre des solutions aux motivations opposées dans une même situation. Le tribunal administratif de Melun avait accepté l'installation de cette crèche au titre d'une motivation longue qu'il convient de reproduire:

Si une crèche peut être regardée comme une reproduction figurative de la naissance de Jésus, elle est dépourvue de toute signification religieuse lorsqu'elle est installée temporairement en dehors des lieux de culte à l'occasion de la fête de Noël et hors de tout contexte rappelant la religion chrétienne, et constitue alors une des décorations traditionnellement associées à Noël comme le sapin de Noël ou les illuminations; qu'en l'espèce, la crèche objet du litige, installée de manière non ostentatoire du 15 au 30 décembre 2012 dans une niche sous le porche permettant de passer de la cour d'honneur de l'Hôtel de ville au jardin public y attenant, et dépourvue de tout autre symbole évoquant la religion chrétienne, doit être regardée comme une des

23. TA Amiens, 17 févr. 2015, Fédération de la libre pensée de l'Oise, $\mathrm{n}^{\mathrm{0}} 1300269$.

24. TA Nantes, 14 nov. 2014, Fédération de Vendée de la libre pensée, $n^{\circ} 1211647$.

25. CAA Nantes, 13 oct. 2015, Département de la Vendée, $n^{\circ}$ 14NTo3400, AJDA 2015, p. 2390 , note Arthur de Dieuleveult. 
décorations festives que la commune a coutume d'installer à l'occasion de Noël; qu'elle ne constitue donc pas un emblème religieux ${ }^{26}$.

Dès lors qu'elle est installée temporairement, et dans un cadre festif, la crèche ne serait plus un signe religieux. La crèche est assimilée à l'ensemble du folklore de Noël et de ce fait est dépourvue de tout caractère religieux en l'absence notamment de toute dimension ostentatoire. La cour administrative d'appel de Paris adopte une solution inverse en décidant qu'une crèche de la nativité doit être invariablement regardée comme un signe religieux ${ }^{27}$. Pour les juges de la cour administrative d'appel de Paris, le contexte ne joue en rien: une crèche représentant la naissance de Jésus est toujours un signe religieux.

Les positions divergentes des juridictions du fond ont plongé les maires dans un embarras profond. Pour tenter de le surmonter l'Association des maires de France a rédigé, en novembre 2015 un vade mecum dans lequel il était affirmé que «la présence de crèches de Noël dans l'enceinte des mairies n'est pas, du point de vue de l'AMF, compatible avec la laïcité» et qu'une clarification législative était souhaitable ${ }^{28}$. La position du Conseil d'État était donc particulièrement attendue, ce qui explique que les deux décisions du 9 novembre $2016^{29}$ aient suscité un flot immédiat et nourri de commentaires ${ }^{30}$.

Il apparaît important, une fois l'émotion retombée, de revenir sur ces deux décisions de principe qui entendaient répondre à la question simple de savoir si une crèche de la nativité pouvait être légalement installée dans l'enceinte d'un bâtiment public. Avec le recul, la complexité du raisonnement demeure. La solution a été qualifiée de «complexe et compliquée ${ }^{31}$ », comme reposant sur « un raisonnement d'une grande

26. TA Melun, 22 déc. 2014, Fédération départementale des libres penseurs de Seine et Marne, $n^{\circ} 1300483$, Dr. adm. 2015, alerte 26, Rozen Noguellou.

27. CAA Paris, 8 oct. 2015, Fédération départementale des libres penseurs de Seine et Marne, $n^{\circ}{ }_{15}$ PAoo814.

28. Maires de France, hors-série, novembre 2015, p. 16.

29. CE, ass., 9 nov. 2016, Fédération départementale de la libre pensée de Seine et Marne, $\mathrm{n}^{\mathrm{O}} 395122$; CE, ass., 9 nov. 2016, Fédération de la libre pensée de Vendée, $\mathrm{n}^{\mathrm{o}} 395223$.

30. Nicolas Chifflot, «Rendre à César ce qui est à César », JCP A 5 déc. 2016, $\mathrm{n}^{\circ} 2309$; Frédéric Rolin, "Y aura-t-il des crèches à Noël? », Dalloz actu, 2016; Matthieu Touzeil-Divina, "Ceci n'est pas une crèche», JCP A 2016, n 853; Alexandre Ciaudo, «Les crèches de Noël dans les bâtiments publics : la messe est dite », Journal $d u$ droit administratif (JDA), 2017, $\mathrm{n}^{\circ} 3$, art 118 ; Jean Morange, « Entre culturel et cultuel», RFDA 2017, p. 127.

31. Nicolas Chifflot, art. cité. 
subtilité, voire d'une certaine artificialité $^{32}$ », tandis que Louis Dutheillet de Lamothe et Guillaume Odinet se demandaient «comment la définition d'un régime juridique aussi complexe pour la seule installation des crèches de Noël a pu paraître opportun à l'Assemblée du contentieux $^{33}$ ». Cette complexité se manifeste de deux manières. D'une part, les décisions abandonnent la simplicité de l'énoncé de l'article 28 de la loi de 1905 pour lui substituer un régime juridique complexe à travers une interprétation finaliste de la loi (1). D’autre part, la portée des solutions retenues demeure incertaine (2).

\section{L'interprétation finaliste de la loi}

Le Conseil d'État fait doublement évoluer le sens de l'article 28 de la loi de 1905. L'interdiction visée par la loi d'apposer tout signe religieux est en effet modifiée en une interdiction des signes reconnaissant une religion, ce qui restreint la portée de l'interdiction (1.1). De plus, les décisions introduisent, de manière totalement prétorienne, une distinction selon que le signe religieux est présent dans un bâtiment servant au service public ou qu'il est apposé sur le domaine public permettant ainsi de nouveau de restreindre la portée de l'interdiction (1.2).

\subsection{Du signe religieux au signe reconnaissant un culte}

L'article 28 de la loi du 9 décembre 1905 pose un principe d'interdiction clair :

Il est interdit, à l'avenir, d'élever ou d'apposer aucun signe ou emblème religieux sur les monuments publics ou en quelque emplacement public que ce soit, à l'exception des édifices servant au culte, des terrains de sépulture dans les cimetières, des monuments funéraires ainsi que des musées ou expositions.

En application de ces dispositions, la jurisprudence considérait ainsi de manière constante que "l'apposition d'un emblème religieux sur un édifice public, postérieurement à l'entrée en vigueur de la loi du 9 décembre 1905 méconnaît la liberté de conscience et la neutralité du service public à l'égard des cultes quels qu'ils soient ${ }^{34}$ ».

32. Gweltaz Eveillard, «Laïcité : crèche de Noël, mode d'emploi... », Dr. adm. avril 2017, comm. 18.

33. Louis Dutheillet de Lamothe et Guillaume Odinet, « La crèche entre dans les tables », AJDA 2016, p. 2375.

34. TA Besançon, 20 déc. 2001, M. Guilleminot c/ Ville de Besançon. 
La loi de 1905 pose donc une interdiction assortie d'exceptions légales. Une exception temporelle d'abord: les signes et emblèmes religieux présents avant l'adoption de la loi peuvent être indéfiniment maintenus. Il n'a donc jamais été question de raser cimetières, églises ou encore calvaires présents de longue date. Des exceptions matérielles ensuite, par l'autorisation de ces signes dans le cadre spirituel, entendu au sens large (édifices servant au culte et à la mémoire des morts) ainsi que dans le cadre culturel (musées et expositions). La place que la religion, et historiquement la religion chrétienne, occupe dans le domaine culturel et social en France était donc déjà pleinement reconnue dans le cadre de la loi de 1905 .

Le principe est clair et suppose que deux qualifications juridiques soient retenues : celle de monument ou d'emplacement public d'abord, celle de signe ou emblème religieux ensuite.

La première qualification ne pose guère de difficultés même si le terme «public» est parfois source de regrettables confusions ${ }^{35}$. Ce n'est pas l'espace public en opposition à la sphère privée qui est visée par le texte, mais uniquement l'espace public au sens de bâtiments du service public ou du domaine public. Autrement dit, la neutralité s'impose dès lors qu'une personne publique est présente. L'exigence de neutralité n'a pas pour finalité de rendre invisibles les signes ou emblèmes religieux, mais bien d'interdire aux personnes publiques, et à elles seules, de les rendre publics. La nuance est fondamentale et nécessaire pour comprendre la portée de l'interdiction. Dans les espèces des arrêts du 9 novembre 2016, la qualification s'impose avec évidence, la nature de monument ou d'emplacement public du hall d'une mairie ou d'un hôtel de département ne faisant guère de doute.

Le second enjeu de qualification juridique est bien plus redoutable et revient à déterminer si un signe ou un emblème est religieux ou non. Ce sujet est extrêmement difficile à deux titres au moins. D'abord, car il est nécessaire de s'interroger sur l'identité de ceux habilités à déterminer la nature religieuse ou non d'un emblème. Il peut sur ce point être objecté que dans un État laïc qui ne reconnaît aucun culte ce ne peut être à l'État de déterminer la nature religieuse ou non d'un emblème ${ }^{36}$. La République ne reconnaissant aucun culte, la République ne voit aucun signe religieux, ne peut en déterminer aucun. Selon cet argument, régulièrement avancé, la soutane du curé ou le voile ne sont que des vêtements et la crèche de la nativité n'est que la représentation

35. Voir, Frédéric Dieu, «Laïcité et espace public », RDP 2013, p. 566.

36. Voir par exemple, David Koussens, op. cit., p. 86. 
figurative d'une famille donnant naissance à un enfant dans une étable. La République étant "aveugle» du point de vue religieux, elle ne peut voir de signe religieux nulle part. Ce raisonnement, séduisant en apparence, empêche alors l'État d'interdire des signes qu'il ne saurait identifier. Mais cela revient alors à vider de sa substance toute la loi de 1905 et notamment son article 28. Quel sens alors aurait l'interdiction des signes religieux si la personne sur laquelle pèse cette obligation, l'État, ne saurait les reconnaitre?

L'identification d'un signe religieux pose aussi difficulté, car un signe ou un emblème peut revêtir une dimension religieuse et en même temps une dimension artistique, culturelle, historique ou patrimoniale. Le Christ de saint Jean de la Croix peint par Salvador Dali est sans conteste une œuvre à la portée culturelle immense. Mais le fait qu'elle représente le Christ en fait-elle un signe religieux, prenant le pas sur sa dimension artistique, et devant, de ce fait, être simplement regardé comme un signe ou un emblème religieux tombant sous le coup de l'interdiction de l'article 28 de la loi de 1905 ? La question est délicate, les exemples pourraient être multipliés.

En l'espèce, cette interrogation, qui ne peut recevoir de réponse définitive, portait sur le fait de savoir si les crèches de la nativité sont ou non un signe religieux. Mais implicitement, et sans que cela n'ait été toujours relevé, le Conseil d'État a modifié la question en se demandant en réalité si une crèche de la nativité est «un signe ou un emblème manifestant la reconnaissance d'un culte ou marquant une préférence religieuse $^{37}$ ». Le glissement est réel et c'est sur ce point que la décision est juridiquement la plus importante. Par là même, la nature de l'obligation imposée aux personnes publiques change.

Cette voie avait déjà été empruntée par le tribunal administratif de Montpellier, également dans une affaire relative aux crèches de la nativité. Le tribunal relevait alors "qu'aucun élément du dossier ne [venait] révéler une intention différente et/ou la manifestation d'une préférence pour les personnes de confession chrétienne, au détriment du reste de la population; qu'ainsi, dans les circonstances de l'espèce, l'installation de cette crèche ne [pouvait] être regardée comme ayant le caractère d'une présentation revendiquée de symboles de la religion chrétienne ${ }^{38} »$.

Le rapporteur public dans ses conclusions prononcées sur les affaires «Fédération de la libre pensée», et qui a donc été suivie sur ce point,

37. Considérant $\mathrm{n}^{\mathrm{o}} 3$.

38. TA Montpellier, 16 juill. 2015, M. D., Ligue des droits de l'homme, $\mathrm{n}^{\mathrm{o}} 1405625$. 
avait également affirmé que le texte n'interdit pas par principe un signe religieux, mais uniquement le signe religieux qui reconnaît l'existence d'un culte. Le signe religieux ne devrait ainsi pas être interdit en tant que tel, mais seulement en raison de l'intention qui l'accompagne. Le changement de paradigme par rapport à 1905 est certain. Ce n'est plus la neutralité du signe qui est exigée, mais seulement l'absence de préférence religieuse. Cela se retrouve dans la formule employée par le Conseil d'État qui pose pour l'État l'obligation de "veiller à la neutralité des agents publics et des services publics à l'égard des cultes, en particulier en n'en reconnaissant aucun ». L'enjeu de l'interdiction ne vise donc pas tant le signe religieux lui-même que sa portée. Ce n'est pas le signe religieux qui est prohibé, mais le signe manifestant la reconnaissance d'un culte ou marquant une préférence religieuse. Ce qui compte donc ce n'est pas l'objet en tant que tel, mais les conditions de son installation. Le Conseil d'État s'inspire ainsi de la jurisprudence de la Cour suprême américaine qui, sur cette question, procède à une analyse contextuelle du signe religieux; celui-ci n'est interdit que s'il constitue un message «d'approbation» de la religion ${ }^{39}$.

On peut considérer que cette nouvelle conception de l'interdiction des signes religieux, en prohibant toute reconnaissance d'un culte et toute préférence religieuse, poursuit la même finalité que la conception classique de la neutralité des services publics à savoir assurer l'égalité des usagers devant le service public. Le changement est toutefois certain et surtout contraire à la lettre de la loi du 9 décembre 1905. Les décisions opèrent également une autre modification du sens de l'article 28 de la loi de 1905 en posant deux régimes juridiques différents selon que le signe religieux est placé dans un bâtiment public ou dans un emplacement public.

\subsection{De l'interdiction absolue à la distinction entre bâtiments et emplacements publics}

L'article 28 de la loi de 1905 interdit les signes ou emblèmes religieux en tous lieux du service public, qu'il s'agisse de "monuments publics » ou de "quelque emplacement public que ce soit». Les décisions du 9 novembre 2016 rompent avec cette unité du régime juridique de l'interdiction pour créer deux régimes distincts, estimant que «la

39. Voir Louis Dutheillet de Lamothe et Guillaume Odinet, art. cité et Thomas Hochmann, "Le Christ, le père Noël et la laïcité en France et aux États-Unis », Nouveaux cahiers $d u$ Conseil constitutionnel, 2016, $\mathrm{n}^{\circ}$ 53, p. 53. 
situation est différente selon qu'il s'agit d'un bâtiment public, siège d'une collectivité publique ou d'un service public, ou d'un autre emplacement public ». Les décisions distinguent donc les bâtiments du service public et le domaine public et créent des régimes juridiques distincts pour chacun d'entre eux.

Il est ainsi affirmé, dans le considérant $\mathrm{n}^{0} 6$ des deux décisions, que «dans l'enceinte des bâtiments publics, sièges d'une collectivité publique ou d'un service public, le fait pour une personne publique de procéder à l'installation d'une crèche de Noël ne peut, en l'absence de circonstances particulières permettant de lui reconnaître un caractère culturel, artistique ou festif, être regardé comme conforme aux exigences qui découlent du principe de neutralité des personnes publiques ».

Concernant les bâtiments publics, le principe de l'interdiction demeure donc. Le Conseil d'État ne fait qu'y apporter une exception bien connue dans la jurisprudence administrative en cas de «circonstances particulières ». Les circonstances particulières de temps et de lieu sont, de longue date, un instrument qu'utilise le juge administratif pour aménager les contours du contrôle de la légalité des mesures de police ${ }^{40}$ ou pour admettre l'activité économique des personnes publiques ${ }^{41}$. L'utilisation de cette notion permet toujours d'atténuer la rigueur d'un principe, ici celui de l'interdiction des signes religieux. Le recours à cette exception ne doit pas étonner et correspond à la démarche finaliste retenue. Il s'agissait en effet de ne pas affecter le principe de neutralité d'une trop grande rigidité et de permettre que des crèches puissent continuer à être installées notamment lorsque cela relève d'une tradition bien établie. Si le mot n'est pas prononcé, c'est bien à la Provence et à ses sentons que les « circonstances particulières» font référence.

Le même principe et la même exception ne se retrouvent pas concernant les emplacements publics autres que les bâtiments publics. Le considérant $\mathrm{n}^{\mathrm{o}} 7$ de ces décisions de principe décide en effet qu' «à l'inverse, dans les autres emplacements publics, eu égard au caractère festif des installations liées aux fêtes de fin d'année notamment sur la voie publique, l'installation à cette occasion et durant cette période d'une crèche de Noël par une personne publique est possible, dès lors qu'elle ne

40. CE, sect. 18 déc. 1959, Société "Les Films Lutetia", Rec. 693, S. 1960.94, concl. Mayras; D. 1960.171, note Prosper Weil, AJDA 1960, I, 21, chron. Combarnous et Galabert; Rev. adm. 1960.31, note Juret.

41. CE, sect., 30 mai 1930, Chambre syndicale du commerce en détail de Nevers, rec. 583, 1931, III, 73, concl. Josse, note Alibert. 
constitue pas un acte de prosélytisme ou de revendication d'une opinion religieuse».

Pour les emplacements publics la solution est donc l'inverse de celle régissant les bâtiments publics: l'installation des crèches de Noël y est possible, sauf si elle revêt le caractère d'un acte prosélyte ou revendicatif d'une opinion religieuse.

Les décisions du 9 novembre créent une distinction entre bâtiments et emplacements publics qui ne se retrouve pas dans la loi du 9 décembre 1905 et instaure « un système de présomptions croisées asymétriques ${ }^{42}$ ». En effet, même si le mot ne figure pas explicitement dans les décisions, ce sont bien des présomptions qui sont posées par le Conseil d'État. Il existe donc dorénavant une présomption d'illégalité de la présence d'une crèche de la nativité dans un bâtiment public sauf si des «circonstances particulières » permettent d'y voir un caractère culturel, artistique ou festif. À l'inverse, dans les emplacements publics, la présence d'une crèche de Noël sera présumée légale sauf si elle est constitutive d'un acte prosélyte ou marquant une préférence religieuse; qui d'ailleurs, concernant un tel signe, ne pourrait concerner que la religion chrétienne.

Cette distinction, véritable obiter dictum, est opportune, mais son fondement juridique demeure particulièrement incertain ${ }^{43}$. En effet, en principe le domaine public lui aussi doit être neutre ${ }^{44}$. Or, selon cette nouvelle jurisprudence de principe, l'installation des crèches de la nativité en fin d'année sera largement admise. Une explication, fragile, peut être avancée à cette différenciation de régime juridique entre bâtiments et emplacements publics, à travers la référence au service public et à l'égalité devant le service public. En effet, il est possible de considérer que celui qui entre dans un bâtiment public et celui qui passe par un emplacement public ne sont pas dans une situation équivalente par rapport au service public, que le lien avec celui-ci, et donc avec ses principes, n'est pas aussi fort dans les deux cas. Celui ou celle qui pousse la porte d'un bâtiment public est un usager du service public et vient y recevoir une prestation (état civil, enseignement, etc.). Comme usager du service public, il est alors légitime que l'ensemble des règles propres au service public, qui ont d'ailleurs pour finalité de le protéger en tant qu'usager, s'applique dans toute leur rigueur. La neutralité, corollaire du principe d'égalité devant le service public, doit donc y être assurée. À l'inverse, le lien entre la personne se trouvant dans un

42. Louis Dutheillet de Lamothe et Guillaume Odinet, art. cité.

43. Gweltaz Eveillard, art. cité.

44. Clément Benelbaz, Le principe de laïcité en droit public français, op. cit., p. 268. 
emplacement public et le service public peut être vu comme moins fort. Certes, l'individu qui se trouve sur la voie publique est usager du service public de la voirie. Toutefois, il n'est pas directement en relation avec un service public, une administration. Par définition le geste d'entrer dans un bâtiment est absent et, partant, la conscience d'entrer en relation avec le service public est sans doute moindre. Pour le dire plus directement, si celui qui entre dans un bâtiment public peut avoir conscience d'être usager du service public cela est beaucoup plus incertain concernant les utilisateurs de la voirie. Cette différence expliquerait alors que les règles inhérentes au fonctionnement du service public ne soient pas aussi fermement appliquées dans les deux hypothèses.

L'explication juridique de cette distinction est bien fragile. On lui préférera donc l'explication pragmatique avancée par Gweltaz Eveillard selon lequel cette solution s'explique par le fait qu'un bâtiment public n'a pas vocation à être festif alors qu'un emplacement public bien davantage ${ }^{45}$. La polysémie de l'espace public, dont il est utilement rappelé qu'il comprend les bâtiments et les espaces publics, donne ainsi lieu à une distinction des régimes juridiques ${ }^{46}$.

Les décisions du 9 novembre 2016 marquent donc deux ruptures avec le texte de la loi de 1905. D'abord en considérant que sont interdits les signes qui reconnaissent l'exercice d'un culte, et non tous les signes religieux. Ensuite, en établissant deux régimes distincts selon que les crèches de Noël sont installées dans les bâtiments ou dans les emplacements publics. On note que, si la première interprétation de la loi de 1905 est générale et vise tous les signes religieux, la seconde interprétation est limitée aux crèches de Noël. Cette différence fait naître le doute sur la portée réelle de ces décisions.

\section{Une portée incertaine}

Les décisions du 9 novembre 2016 constituent une lecture plus souple, plus nuancée et parfois présentée comme plus contemporaine, des principes de la loi de 1905, en refusant d'interdire de manière générale et absolue les crèches de Noël dans les bâtiments publics. À la lecture de ces décisions, l'incertitude demeure toutefois sur le fondement de cette solution. L'installation des crèches est-elle possible en raison de leur absence de caractère religieux ou en dépit de celui-ci ? (2.1). De plus,

45. Gweltaz Eveillard, art. cité.

46. Frédéric Dieu, art. cité. 
une incertitude demeure sur la portée de cette solution dont on peut se demander si elle est circonscrite aux seules crèches de Noël (2.2).

\subsection{Un fondement incertain}

Un des éléments essentiels conditionnant la solution retenue par le Conseil d'État tenait à la qualification d'une crèche de Noël comme signe ou emblème religieux. Dans son considérant $\mathrm{n}^{\mathrm{o}} 4$, le Conseil d'État décide "qu'une crèche de Noël est une représentation susceptible de revêtir une pluralité de significations ". "Il s'agit en effet d'une scène qui fait partie de l'iconographie chrétienne et qui, par là, présente un caractère religieux. Mais il s'agit aussi d'un élément faisant partie des décorations et illustrations qui accompagnent traditionnellement, sans signification religieuse particulière, les fêtes de fin d'année. »

La pluralité de significations que revêtent ces crèches en fait donc, non pas successivement, mais en même temps, un signe religieux et un signe apposé «sans signification religieuse particulière». Au regard de ce qui a pu être décidé par ailleurs, on peut donc comprendre que si une crèche de Noël est un signe religieux ce signe ne porte pas nécessairement reconnaissance d'un culte.

Cette lecture est confirmée au considérant $\mathrm{n}^{0} 5$, élément central du raisonnement et du dénouement. Il y est en effet décidé qu' « eu égard à cette pluralité de significations, l'installation d'une crèche de Noël, à titre temporaire, à l'initiative d'une personne publique, dans un emplacement public, n'est légalement possible que lorsqu'elle présente un caractère culturel, artistique ou festif, sans exprimer la reconnaissance d'un culte ou marquer une préférence religieuse». "Pour porter cette dernière appréciation, il y a lieu de tenir compte non seulement du contexte, qui doit être dépourvu de tout élément de prosélytisme, des conditions particulières de cette installation, de l'existence ou de l'absence d'usages locaux, mais aussi du lieu de cette installation.»

La lecture minutieuse de l'arrêt révèle donc que trois conditions sont exigées pour qu'une crèche puisse être légalement installée : le caractère temporaire de l'installation, son caractère culturel, artistique ou festif et l'absence de préférence religieuse. La première condition ne pose guère de difficultés tant elle est consubstantielle au sujet en cause et que l'on imagine mal l'installation d'une crèche à un autre moment de l'année. La seconde condition n'est en revanche pas explicitée alors que le caractère "culturel, artistique ou festif» d'une installation ne semble pas nécessairement aller de soi. Cela signifie-t-il que toute exposition pourrait accueillir des signes religieux et particulièrement 
des crèches de Noël ? Qu'une fête des religions pourrait être organisée dans un bâtiment public? Ces questions ne sont pas tranchées et sont laissées à l'appréciation ultérieure des juridictions administratives. En revanche, le Conseil d'État précise que la dernière condition relative à l'absence de prosélytisme doit être appréciée au regard des conditions particulières de l'installation, de son lieu ou de l'absence d'usages locaux. Ces conditions posées pour reconnaître la légalité de l'installation des crèches de Noël sont beaucoup plus rigoureuses que celles qu'avait suggérées le rapporteur public et qui auraient conduit à reconnaitre la légalité de l'installation de la plupart des crèches.

Par un raisonnement habile, et parfois circulaire, le Conseil d'État réussit en définitive à éviter d'apporter une réponse à la question initiale et essentielle pour la solution du litige: la crèche est-elle un signe religieux?

Cette question semble pourtant devoir recevoir une réponse évidente : la crèche de la nativité est la représentation figurative de la naissance de Jésus qui, pour l'ensemble des chrétiens, soit plus de 2 milliards de personnes dans le monde, est fils de Dieu et Dieu lui-même. Si on ne reconnaît pas à une représentation de la naissance de Dieu le caractère d'un signe religieux alors on conçoit mal quels emblèmes pourraient être ainsi qualifiés. La crèche est ontologiquement, par sa nature même, un signe religieux ${ }^{47}$. Le fait qu'il y ait une pluralité de sens à la crèche, une "surcouche" culturelle selon l'expression de Frédéric Rolin, ne fait pas pour autant disparaître sa dimension religieuse, elle ne l'efface pas ${ }^{48}$. L'éventuelle dimension culturelle de l'objet n'a pas pour effet de faire disparaître la dimension religieuse que l'on pourrait qualifier de nécessairement première. Comme le remarque Thomas Hochmann, «même entouré d'un renne et d'un sapin [...] la signification religieuse de la crèche ne saurait être éliminée ${ }^{49}$ ». Dans le même sens, Mathieu Touzeil-Divina relève que «ce n'est donc pas parce que la crèche est aujourd'hui également une expression culturelle et sociétale non religieuse pour de nombreux citoyens que cela gomme cet élément culturel originel ». La pluralité de sens n'a pas pour effet de faire disparaître chacun d'eux : « Le fait qu'il y ait une pluralité indéniable de sens à un symbole religieux ne fait pas disparaître le religieux. Au pire elle le dilue mais ne l'efface pas ${ }^{50}$.» Enfin, Philippe Cossalter rappelle

47. Nicolas Chifflot, art. cité.

48. Frédéric Rolin, art. cité.

49. Thomas Hochmann, art. cité., p. 53.

50. Matthieu Touzeil-Divina, art. cité. 
que les promoteurs de la crèche comme élément culturel en sont pour leurs frais, car cela revient à reléguer un élément de la foi chrétienne, que certains entendent défendre par l'installation de crèches, à un simple élément de folklore comme le sapin ou la bûche ${ }^{51}$.

La formulation retenue par le Conseil d'État est ambiguë. Elle reconnaît que la crèche n'est pas uniquement un signe religieux, mais elle ne prend pas directement parti sur le fait de savoir si elle peut être considérée comme n'étant pas un signe religieux. Le Conseil d'État semble donc admettre que la crèche de la nativité n'a pas nécessairement de sens religieux même si elle demeure toujours un signe religieux. L'ambiguiité demeure quant au champ d'application de ces décisions qui demeure assez largement incertain.

\subsection{Un champ d'application potentiellement incertain}

La portée de ces décisions de principe doit avant tout être mesurée au regard des applications d'espèce. Dans l'affaire de la crèche dans le hall de l'hôtel de département de la Vendée le Conseil d'État décide habilement de ne pas juger au fond. Il laisse donc le soin à la cour administrative d'appel de Nantes de décider si la présence de cette crèche ressortait d'un usage local. La question était ici plus délicate que dans l'affaire de Melun, car la crèche est installée depuis près de trente ans dans ce bâtiment; de plus dans un département où les usages aux références religieuses, catholiques précisément, sont peutêtre légèrement différents et où il existe peut-être des «circonstances particulières ${ }^{52} »$. La cour administrative d'appel de Nantes a d'ailleurs admis la légalité de l'installation de cette crèche ${ }^{53}$. Elle s'est pour cela fondée sur plusieurs critères: le temps depuis lequel il est habituel que cette crèche soit installée en fin d'année (depuis 1990), les dates de son installation ( $\mathrm{du}_{1}{ }^{\mathrm{er}}$ décembre au 10 janvier, dates «exemptes de toute tradition ou référence religieuse), sa taille ( $3 \mathrm{~m}$ sur $2 \mathrm{~m}$ au sein d'un hall de plus de 1 ooo $\mathrm{m}^{2}$ ). Au regard de l'ensemble de ces éléments la cour conclut donc à l'absence de prosélytisme et que dans ces conditions particulières l'installation de la crèche « résulte d'un usage culturel local et d'une tradition festive». L'application des solutions de principe aux

51. Philippe Cossalter, «Pour rester un symbole chrétien la crèche doit être exclue de l'espace public », Revue générale du droit, 2016, $\mathrm{n}^{\circ} 23432$. Voir également, Nicolas Chifflot, art. cité.

52. À propos du logo du conseil général de Vendée: CAA Nantes, 11 mars 1999, Association « une Vendée pour tous les Vendéens ", $\mathrm{n}^{\circ}$ 98NTo0357.

53. CAA, Nantes, 6 oct. 2017, $\mathrm{n}^{\circ} 16 \mathrm{NT} 03735$. 
espèces concernées confirme le caractère extrêmement concret du juge en la matière.

Dans l'affaire relative à la crèche de l'hôtel de ville de Melun, le Conseil d'État estime que cette installation est contraire à l'article 28 de la loi de 1905, car réalisée «en l'absence de circonstances particulières permettant de lui reconnaître un caractère culturel, artistique ou festif ». Cette motivation est intéressante à double titre. D'une part, elle adopte une conception très restrictive de l'usage local en en déniant l'existence en l'espèce. D'autre part, elle confirme que le simple fait d'être installée pendant la période de Noël ne suffit pas à donner à cette crèche un caractère "culturel, artistique ou festif». Ces solutions doivent donc être lues à la lumière de l'application d'espèce pour en apprécier justement la portée. Il en ressort clairement qu'en définitive le principe d'interdiction des signes religieux n'est pas vidé de sa substance et qu'à l'exception des cas dans lesquels des circonstances particulières seront incontestablement constatées, il sera toujours impossible d'ériger des crèches de Noël dans des bâtiments publics sièges des services publics.

La décision doit être comprise au-delà de l'affirmation affichée selon laquelle l'installation des crèches est possible sous condition. Il faut en réalité comprendre que les crèches sont interdites sauf exception. Et ces exceptions semblent devoir être fort limitées. Par les applications d'espèce le Conseil d'État restreint donc le champ d'application de cette jurisprudence que l'on pourrait qualifier de « jurisprudence spécial santons de Provence» tant les termes des considérants de principe que l'application qui en est faite peuvent laisser croire qu'il s'agit avant tout de protéger cette spécificité régionale.

Les quelques décisions rendues par la suite par les juridictions du fond confirment cette portée très restrictive des décisions du 9 novembre. Le 30 novembre 2016 , le tribunal administratif de Lille avait à connaitre de la légalité de l'installation d'une crèche de la nativité dans les locaux de la commune d'Hénin-Beaumont ${ }^{54}$. La décision reprend le considérant de principe en l'amendant légèrement:

Le fait pour une personne publique de procéder à l'installation d'une crèche de Noël ne peut, en l'absence de circonstances particulières permettant de lui reconnaître un caractère culturel, artistique ou festif, être regardé comme conforme aux exigences qui découlent du principe de neutralité des personnes publiques.

54. TA Lille, 30 nov. $2016, \mathrm{n}^{\circ} 1509979$. 
Le tribunal relève que «ladite crèche composée de sujets sans valeur historique ou artistique particulière a revêtu le caractère d'une exposition d'œuvres d'art» et que «les sujets ne sont en rien liés à une tradition minière spécifique » et qu'il n'est pas établi «qu'elle s'enracine dans une tradition locale préexistante » sans qu'il y ait alors besoin d'examiner le caractère ostentatoire ou prosélyte de cette crèche. Le caractère très restrictif des exceptions à l'interdiction est confirmé.

Dans une autre affaire, la cour administrative d'appel de Marseille annule le jugement du tribunal administratif de Montpellier qui n'avait pas déclaré illégale l'installation de la crèche dans le hall de la commune de Béziers ${ }^{55}$. Là encore les usages locaux autorisant une telle installation sont entendus avec une grande rigueur et ne sont pas reconnus en l'espèce. Enfin, le tribunal administratif de Lyon a décidé, par deux décisions du 5 octobre 2017, que l'installation d'une crèche de taille imposante dans le hall d'entrée de l'hôtel de région Auvergne-RhôneAlpes était illégale ${ }^{56}$. En effet, une telle crèche ne résultait d'aucun usage local, le tribunal précisant qu' 'aucune crèche de Noël n'a jamais été installée dans les locaux du siège lyonnais de la région Auvergne-RhôneAlpes ».

La portée particulière de ces décisions semble donc aujourd'hui poursuivre une voie étroite qui limite fortement les divergences par rapport à la lettre et à l'esprit de la loi de 1905. En revanche, la portée générale de ces décisions demeure encore incertaine. En effet, de manière étonnante, les considérants de principe de ces décisions ne font état que d'une espèce particulière: les crèches de Noël. La question se pose donc légitimement de savoir si cette solution s'applique à l'ensemble des signes religieux ou si elle est circonscrite aux seules crèches de Noël. Ainsi, on ne sait si, par exemple, pour la fête juive de Soukkot des tentes culturelles, artistiques et festives pourraient être acceptées ou si pour la fête de l'Aïd une manifestation culturelle, artistique et festive pourrait se tenir dans un bâtiment public. Au regard du contexte de la décision et de la motivation retenue, on peut légitimement penser que le Conseil d'État a entendu limiter la portée de ces décisions au seul cas des crèches de Noël. Gweltaz Eveillard relève d'ailleurs que le raisonnement tenu n'est pas nécessairement transposable à d'autres signes religieux

55. CAA Marseille, 3 avr. 2017, David G. et Ligue des droits de l'homme, $\mathrm{n}^{\mathrm{o}}{ }_{15 \mathrm{MA0}} 863$, JCP A 2017, $\mathrm{n}^{\mathrm{o}}$ 16, p. 9, obs. Mathieu Touzeil-Divina.

56. TA Lyon, 5 oct. 2017, Fédération de la libre pensée et d'action sociale du Rhône, $\mathrm{n}^{\mathrm{o}}$ 1701752; TA Lyon, 5 oct. 2017, Ligue française pour la défense des droits de $l^{\prime}$ Homme et du citoyen, $n^{\circ} 1609063$. 
en raison de la spécificité du lien quasi indissociable entre culturel et cultuel concernant les crèches de Noë ${ }^{57}$. On retrouve alors l'ambigüité de la distinction entre le culturel et le cultuel qui amène à ne pas traiter l'ensemble des signes religieux de manière identique. Toutefois, c'est bien de manière générale que le Conseil d'État pose l'interdiction «d'un signe ou emblème manifestant la reconnaissance d'un culte ou marquant une préférence religieuse ${ }^{58} »$. Sur ce point, le changement fondamental de conception semble devoir concerner l'ensemble des signes religieux. En cela, ces décisions entrouvrent une porte qui peut laisser place à de nombreuses difficultés. D'autres signes religieux que les crèches pourront en effet être apposés au motif, bien difficile à établir, qu'il ne s'agit pas de reconnaître un culte.

La dimension politique et symbolique de ces affaires ne peut être ignorée, pas plus que ne peut être ignoré le fait que ces dimensions extrajuridiques ont contraint le Conseil d'État dans la solution et la motivation retenues. En cela, il s'agit effectivement d'une « jurisprudence pragmatique et soucieuse de la paix sociale ${ }^{59} »$. On ne peut toutefois pas non plus éluder le fait qu'une brèche est ouverte dans le principe de la neutralité des services publics qui, rappelons-le, ne concerne pas uniquement la neutralité religieuse. Or, l'exigence de neutralité ne peut qu'être absolue. Un service est neutre ou ne l'est pas. Il ne peut y avoir, par définition, de voie médiane. La neutralité impose donc l'absence de tout signe distinctif, de quelque nature que ce soit. D'ailleurs, au-delà même des principes, les bâtiments publics ne sont pas le lieu adéquat pour y apposer un signe religieux, une propagande commerciale ou politique ou les marques de l'attachement à un club sportif particulier. Les bâtiments publics doivent se limiter à être le lieu du service public, ce qui est déjà beaucoup.

Maître de conférences en droit public Université de Rouen, CUREJ (EA 4703)

57. Gweltaz Eveillard, art. cité.

58. Cette grille d'analyse a d'ailleurs été reprise pour juger illégale la croix surmontant la statue représentant Jean-Paul II sur la place d'une commune. CE, 25 oct. 2017, Fédération morbihannaise de la libre pensée et autres, $\mathrm{n}^{\mathrm{O}} 396990$.

59. Matthieu Touzeil-Divina, JCP A 2017, $\mathrm{n}^{\circ}$ 16, p. 10. 